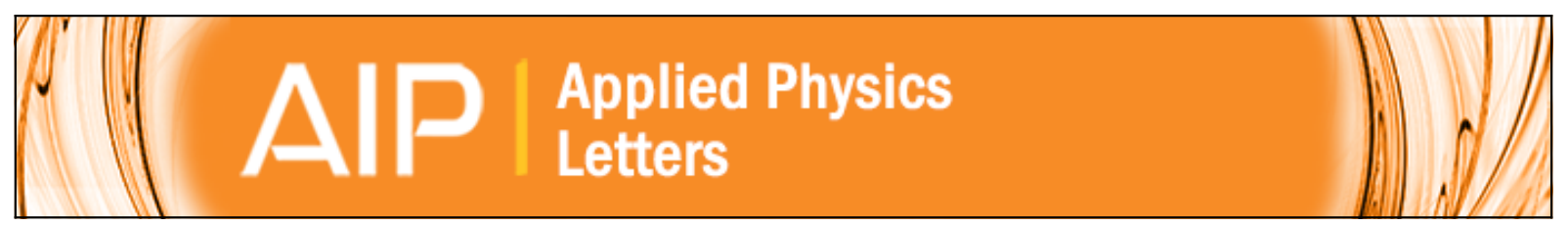

\title{
Magnetic and transport properties of epitaxial stepped Fe304(100) thin films
}

Han-Chun Wu, Askar Syrlybekov, Ozhet Mauit, Anas Mouti, Cormac Ó Coileáin, Mourad Abid, Mohamed Abid, and Igor V. Shvets

Citation: Applied Physics Letters 105, 132408 (2014); doi: 10.1063/1.4897001

View online: http://dx.doi.org/10.1063/1.4897001

View Table of Contents: http://scitation.aip.org/content/aip/journal/apl/105/13?ver=pdfcov

Published by the AIP Publishing

\section{Articles you may be interested in}

Pillar shape modulation in epitaxial BiFeO3-CoFe2O4 vertical nanocomposite films

APL Mat. 2, 081101 (2014); 10.1063/1.4892695

Origin of the twofold and fourfold symmetric anisotropic magnetoresistance in epitaxial Fe 304 films

J. Appl. Phys. 108, 093921 (2010); 10.1063/1.3499696

Fourfold symmetric anisotropic magnetoresistance based on magnetocrystalline anisotropy and antiphase boundaries in reactive sputtered epitaxial Fe 304 films

Appl. Phys. Lett. 96, 092502 (2010); 10.1063/1.3334722

Surface magnetic structure of epitaxial magnetite thin films grown on $\mathrm{MgO}(001)$

J. Appl. Phys. 105, 07 D545 (2009); 10.1063/1.3089493

Magnetotransport properties of Fe 304 epitaxial thin films: Thickness effects driven by antiphase boundaries J. Appl. Phys. 100, 103902 (2006); 10.1063/1.2386927

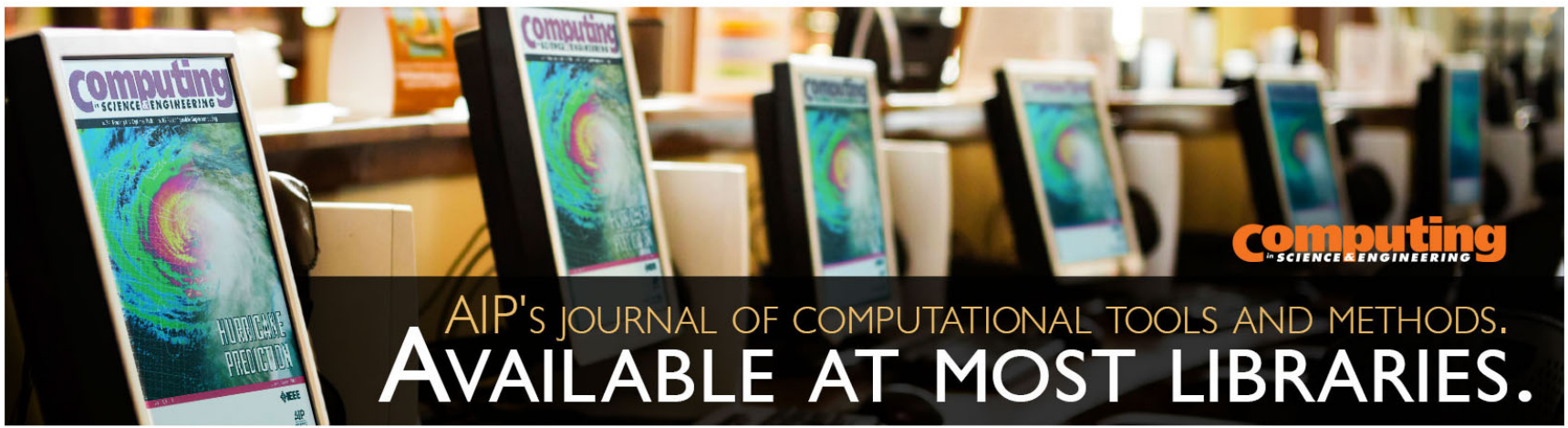




\title{
Magnetic and transport properties of epitaxial stepped $\mathrm{Fe}_{3} \mathrm{O}_{4}(100)$ thin films
}

\author{
Han-Chun Wu, ${ }^{1,2, a)}$ Askar Syrlybekov, ${ }^{3}$ Ozhet Mauit, ${ }^{3}$ Anas Mouti, ${ }^{4}$ Cormac Ó Coileáin, ${ }^{2,3}$ \\ Mourad Abid, ${ }^{2}$ Mohamed Abid, ${ }^{2, a)}$ and Igor V. Shvets ${ }^{3}$ \\ ${ }^{1}$ School of Physics, Beijing Institute of Technology, Beijing 100081, People's Republic of China \\ ${ }^{2}$ KSU-Aramco Center, King Saud University, Riyadh 11451, Saudi Arabia \\ ${ }^{3}$ CRANN, School of Physics, Trinity College, Dublin 2, Ireland \\ ${ }^{4}$ Materials Science \& Technology Division, Oak Ridge National Laboratory, Oak Ridge, \\ Tennessee 37831, USA
}

(Received 22 August 2014; accepted 20 September 2014; published online 1 October 2014)

\begin{abstract}
We investigate the magnetic and transport properties of epitaxial stepped $\mathrm{Fe}_{3} \mathrm{O}_{4}$ thin films grown with different thicknesses. Magnetization measurements suggest that the steps induce additional anisotropy, which has an easy axis perpendicular to steps and the hard axis along the steps. Separate local transport measurements, with nano-gap contacts along a single step and perpendicular to a single step, suggest the formation of a high density of anti-phase boundaries (APBs) at the step edges are responsible for the step induced anisotropy. Our local transport measurements also indicate that APBs distort the long range charge-ordering of magnetite. (C) 2014 AIP Publishing LLC.

[http://dx.doi.org/10.1063/1.4897001]
\end{abstract}

Ferromagnetic thin films grown epitaxially on vicinal substrate surfaces have attracted much attention recently due to their interesting magnetic and transport properties, ${ }^{1-12}$ and potential for applications in spintronics. ${ }^{13}$ Most studies focus on anisotropy induced by step arrays, particularly metallic films on stepped surfaces, such as Fe on stepped $\mathrm{Ag},{ }^{2-4} \mathrm{Au},{ }^{4}$ $\mathrm{W},{ }^{5-7}$ and $\mathrm{Si},{ }^{12} \mathrm{Fe}_{1-\mathrm{x}} \mathrm{Co}_{\mathrm{x}}$ on stepped $\mathrm{GaAs},{ }^{8} \mathrm{Co}$ on stepped $\mathrm{Cu},{ }^{9,10}$ and $\mathrm{CoPt}_{3}$ on stepped $\mathrm{MgO} .{ }^{11}$ However, the alignment of the magnetization easy axis, associated with this step induced anisotropy, is debatable. It is sometimes parallel to the step edges and at other times perpendicular to them. Magnetite, $\mathrm{Fe}_{3} \mathrm{O}_{4}$, is an important transition metal oxide with a nearly fully spin polarized electron band at the Fermi level (half-metallic character) and a high Curie temperature of $858 \mathrm{~K}$, which make it a promising candidate for room temperature spintronic devices and applications. ${ }^{14-16}$ Recently, interesting magnetic and transport properties in epitaxial $\mathrm{Fe}_{3} \mathrm{O}_{4}$ have been reported, i.e., magnetism in nanometerthick magnetite, ${ }^{17}$ large orbital moment in nanoscale magnetite, ${ }^{18}$ giant magnetization in nanometer-thick magnetite, ${ }^{19}$ a spin Seebeck effect, ${ }^{20}$ a spin filter effect, ${ }^{21}$ electrical fieldinduced phase transition, ${ }^{22-24}$ large transversal magnetoresitance (MR), ${ }^{25,26}$ and spin valve effect. ${ }^{27-29}$ However, initial efforts in exploiting its half metallic nature in magnetic tunnel junctions (MTJ) have been far from promising. ${ }^{28,29}$ The presence of anti-phase boundary defects in $\mathrm{Fe}_{3} \mathrm{O}_{4}$ contribute to its unusual magnetic and transport properties, such as the magnetization non-saturation even at very high field, ${ }^{30,31}$ the super-paramagnetic behavior in $\mathrm{Fe}_{3} \mathrm{O}_{4}$ films, ${ }^{32,33}$ and a greater MR response across the AF-APBs. ${ }^{34-36}$ On the other hand, in polycrystalline $\mathrm{Fe}_{3} \mathrm{O}_{4}$ thin films, interesting properties such as magnetic-transport, ${ }^{37}$ spin-injection, ${ }^{38}$ and charge ordering ${ }^{39,40}$ have also been investigated and discussed. Therefore, it is worth investigating the magnetic properties of stepped epitaxial $\mathrm{Fe}_{3} \mathrm{O}_{4}$ films produced on large

\footnotetext{
${ }^{\text {a) }}$ Authors too whom correspondence should be addressed: Electronic addresses: wuhc@tcd.ie and moabid@ksu.edu.sa
}

miscut angle vicinal $\mathrm{MgO}$ substrates, as a high density APBs is expected along the step edges. Moreover, this arrangement could be useful to help understand the effects of APBs on the conduction mechanisms within $\mathrm{Fe}_{3} \mathrm{O}_{4}$ and its metal to insulator transition (Verwey transition).

This letter describes how we fabricated epitaxial stepped $\mathrm{Fe}_{3} \mathrm{O}_{4}$ thin films of different thicknesses and provides details of their magnetic and transport properties. The morphology of $\mathrm{Fe}_{3} \mathrm{O}_{4}$ thin films was characterized by atomic force microscopy (AFM) and high resolution scanning transmission electron microscopy (STEM). The M (H) measurements suggest that the steps induce additional anisotropy which has its easy axis perpendicular to steps and the hard axis along the steps. Local transport measurements suggest that the step induced anisotropy is mainly due to APBs formed at the step edges.

To grow stepped $\mathrm{Fe}_{3} \mathrm{O}_{4}$ films, the first step is to prepare a stepped $\mathrm{MgO}$ (100) substrate. In this work, single-side polished $\mathrm{MgO}$ (100) single crystalline substrates with a miscut angle of $2.8^{\circ}$ in the $\langle 001\rangle$ direction were used. The steps were produced by annealing the substrates in air in a furnace at high temperature. The terrace width and height of the steps were tuned by selection of the substrate annealing temperature and time. To produce uniform, regular, and straight step array on the $\mathrm{MgO}$ surface, the $\mathrm{MgO}$ substrates were annealed at $1300{ }^{\circ} \mathrm{C}$ for $3 \mathrm{~h}$. Figure 1(a) shows a typical AFM image of a stepped $\mathrm{MgO}$ substrate. Uniform, regular, and straight steps can be clearly observed, and the steps are parallel to the $\langle 010\rangle$ direction. The average terrace width for this substrate is around $160 \mathrm{~nm} . \mathrm{Fe}_{3} \mathrm{O}_{4}$ films with thickness of 5, 10, 15, and $20 \mathrm{~nm}$ were grown on stepped $\mathrm{MgO}$ substrates using oxygen plasma assisted molecular beam epitaxy (MBE) with a base pressure of $3 \times 10^{-10}$ Torr. To ensure that the growth conditions were the same for the different thickness films, the $\mathrm{Fe}_{3} \mathrm{O}_{4}$ layers were grown on the same $\mathrm{MgO}$ substrate using a shadow mask inside the MBE system. Several additional films with a thickness of $60 \mathrm{~nm}$ were also prepared for electrical measurements. After their annealing in air to produce the 

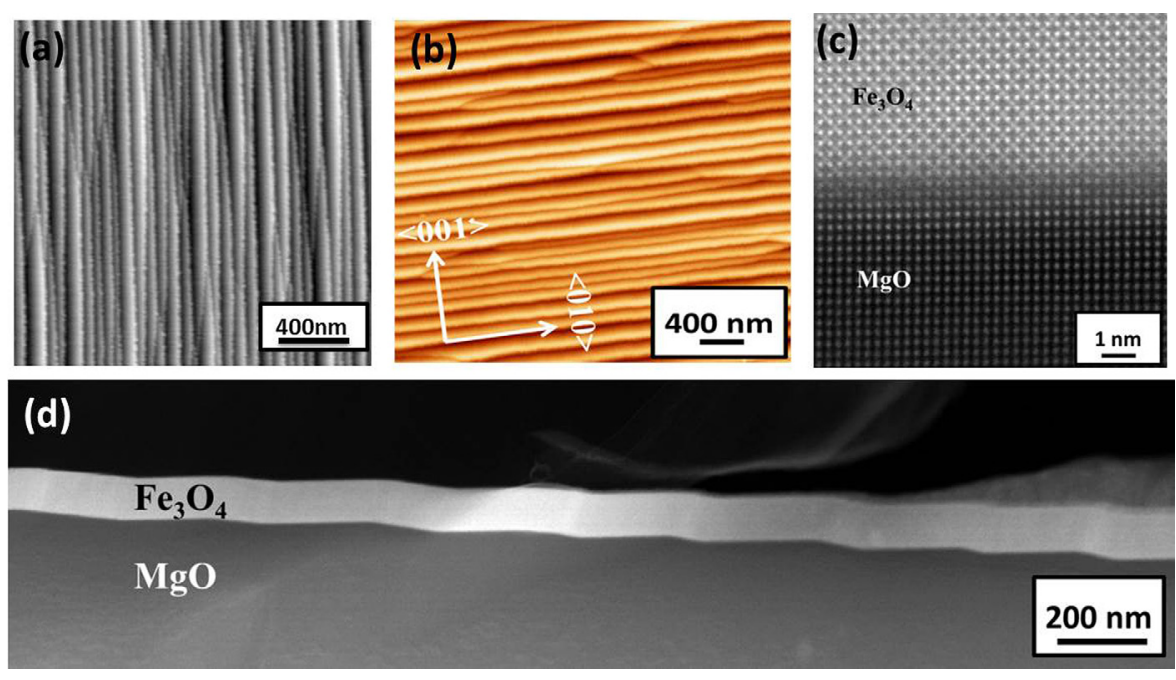

FIG. 1. (a) An AFM image of a $\mathrm{MgO}$ substrate with a miscut angle of $2.8^{\circ}$ annealed at $1300^{\circ} \mathrm{C}$ for $3 \mathrm{~h}$. The steps are parallel to the $\langle 010\rangle$ direction. (b) A $4 \times 4 \mu \mathrm{m}^{2}$ AFM image of $60 \mathrm{~nm}$ thick $\mathrm{Fe}_{3} \mathrm{O}_{4}$ deposited on a stepped $\mathrm{MgO}$ substrate. The steps are parallel to the $\langle 010\rangle$ direction. (c) High resolution STEM image indicating epitaxial growth of the $\mathrm{Fe}_{3} \mathrm{O}_{4}$ on the stepped $\mathrm{MgO}$ substrate. (d) Low magnification STEM image demonstrating the epitaxial growth of stepped $\mathrm{Fe}_{3} \mathrm{O}_{4}$. steps, the substrates were chemically cleaned prior to insertion into the growth chamber and were further annealed at $600{ }^{\circ} \mathrm{C}$ in UHV for $30 \mathrm{~min}$ and subsequently annealed in oxygen at a partial pressure of $1.3 \times 10^{-5}$ Torr for $2 \mathrm{~h}$. The $\mathrm{Fe}_{3} \mathrm{O}_{4}$ films were grown by electron beam evaporation of metallic iron $(99.999 \%)$ in the presence of free oxygen radicals generated by an electron cyclotron resonance plasma source with a growth rate of $0.3 \AA / s$. The substrate temperature during growth was kept at $250{ }^{\circ} \mathrm{C}$. Reflection high energy electron diffraction (RHEED) was employed to confirm the epitaxial growth and establish the growth mode. Details of the film growth is given elsewhere. ${ }^{19,26,27}$ Figure 1(b) shows a $4 \times 4 \mu \mathrm{m}^{2}$ AFM image of $60 \mathrm{~nm}$ thick $\mathrm{Fe}_{3} \mathrm{O}_{4}$ deposited on a stepped $\mathrm{MgO}$ substrate. It is clear the film keeps the morphology of the substrate. The steps remain parallel to the $\langle 010\rangle$ direction, and the terrace width and height of the steps are around $160 \mathrm{~nm}$ and $8 \mathrm{~nm}$, respectively. To further demonstrate the stepped structure of the $\mathrm{Fe}_{3} \mathrm{O}_{4}$ films, we show in Fig. 1(d) a low-magnification high angle angular dark field (HAADF) STEM image of the whole depth of the $\mathrm{Fe}_{3} \mathrm{O}_{4}$ thin film (bright) on the $\mathrm{MgO}$ substrate (dark). One can clearly see that the $\mathrm{Fe}_{3} \mathrm{O}_{4}$ films were epitaxially grown on the $\mathrm{MgO}$ substrates and the $\mathrm{Fe}_{3} \mathrm{O}_{4}$ thin films do have a stepped character. One can also see from Fig. 1(d) that the interface between $\mathrm{Fe}_{3} \mathrm{O}_{4}$ and $\mathrm{MgO}$ is coherent and defect free. We also show a high resolution STEM image in Fig. 1(c), which further demonstrates the epitaxial nature of the films even at the step edges.

Figure 2 shows $\mathrm{M}(\mathrm{H})$ loops of stepped $\mathrm{Fe}_{3} \mathrm{O}_{4}$ thin films measured at room temperature using a Physical Property Measurement System (PPMS) (Quantum Design) equipped with a $14 \mathrm{~T}$ superconducting magnet. The magnetic field was applied in-plane along the steps (AL), i.e., along $\langle 010\rangle$ and perpendicular to the steps (PS). The room temperature saturation magnetizations for the $5,10,15$, and $20 \mathrm{~nm}$ thick stepped $\mathrm{Fe}_{3} \mathrm{O}_{4}$ film are $450,465,475$, and $480 \mathrm{emu} / \mathrm{cm},{ }^{3}$ respectively, which are less than the bulk value. The reduced saturation magnetization is due to the presence of antiferromagnetic the anti-phase boundaries formed at the step edges. ${ }^{33}$ A significant difference between $\mathrm{M}(\mathrm{H})$ loops for the orthogonal field directions can be clearly observed in Fig. 2. For the $5 \mathrm{~nm}$ $\mathrm{Fe}_{3} \mathrm{O}_{4}$ film with the field perpendicular to the steps, the coercivity field is 65 Oe. While for the field along the steps, the coercivity field is only 33 Oe. Moreover, the M (H) loop for the field perpendicular to the steps is much squarer than for field applied along steps. Similar behavior was observed for all the films. It is clear that the easy axis, for all the films grown, is perpendicular to the steps and hard axis is along the steps. We can also see from Fig. 2 that, with increasing film thickness, the difference in the $\mathrm{M}(\mathrm{H})$ loops for two field directions decreases. In contrast to stepped samples, the inset of Fig. 2(a) shows $\mathrm{M}(\mathrm{H})$ _loops of a $5 \mathrm{~nm} \mathrm{Fe} \mathrm{O}_{4}$ film without steps, fabricated with the same conditions. There is no discernable difference between the $\mathrm{M}(\mathrm{H})$ loops for the two field directions. The $\mathrm{M}(\mathrm{H})$ measurements on stepped $\mathrm{Fe}_{3} \mathrm{O}_{4}$ thin films were also performed at a variety of temperatures. Figures 3(a)-3(e) show M (H) loops of $20 \mathrm{~nm}$ thick stepped $\mathrm{Fe}_{3} \mathrm{O}_{4}$ thin films acquired at different temperatures. Figure 3(f) shows the magnetization vs temperature (M-T) curves measured during the heating cycle at a field of 200 Oe applied for both orthogonal field directions. At around $115 \mathrm{~K}$, the Verwey transition is clearly present, which further indicates the epitaxial nature of the stepped $\mathrm{Fe}_{3} \mathrm{O}_{4}$ film. There is a small difference in the M-T curves measured for both field directions which can attributed to different initial states as the coercivity field below the Verwey transition temperature is larger than 200 Oe. Nerveless the coercivity field of the film increases significantly across the Verwey transition and steplike features in the $\mathrm{M}(\mathrm{H})$ loops appear below the Verwey transition temperature, similar behavior was observed at all temperatures. The easy axis of the film is perpendicular to the steps and hard axis is along the steps even below the Verwey temperature. The step-like features in the $\mathrm{M}(\mathrm{H})$ loops observed in Figs. 3(e) and 3(f) are mainly due to the proximity effect of anti-phase boundaries. ${ }^{41}$ The difference in the $\mathrm{M}$ (H) loops can be understood by considering the preferential formation of APBs in $\mathrm{Fe}_{3} \mathrm{O}_{4}$ films during growth on stepped surfaces. $\mathrm{Fe}_{3} \mathrm{O}_{4}$ has a cubic inverse spinel structure, where $\mathrm{Fe}^{3+}$ ions occupy tetrahedral sites (A-sites) and octahedral sites (B-sites) are occupied by both $\mathrm{Fe}^{3+}$ and $\mathrm{Fe}^{2+}$ ions. The symmetry $(\mathrm{Fd} 3 \mathrm{~m})$ of $\mathrm{Fe}_{3} \mathrm{O}_{4}$ is lower than that of $\mathrm{MgO}$ $(\mathrm{Fm} 3 \mathrm{~m})$ and the lattice parameter of $\mathrm{Fe}_{3} \mathrm{O}_{4}(8.397 \AA)$ is almost twice of that of the $\mathrm{MgO}$ substrate (4.213 $\AA$ ). Thus, APBs will form when growing $\mathrm{Fe}_{3} \mathrm{O}_{4}$ on $\mathrm{MgO}$ and there is a 

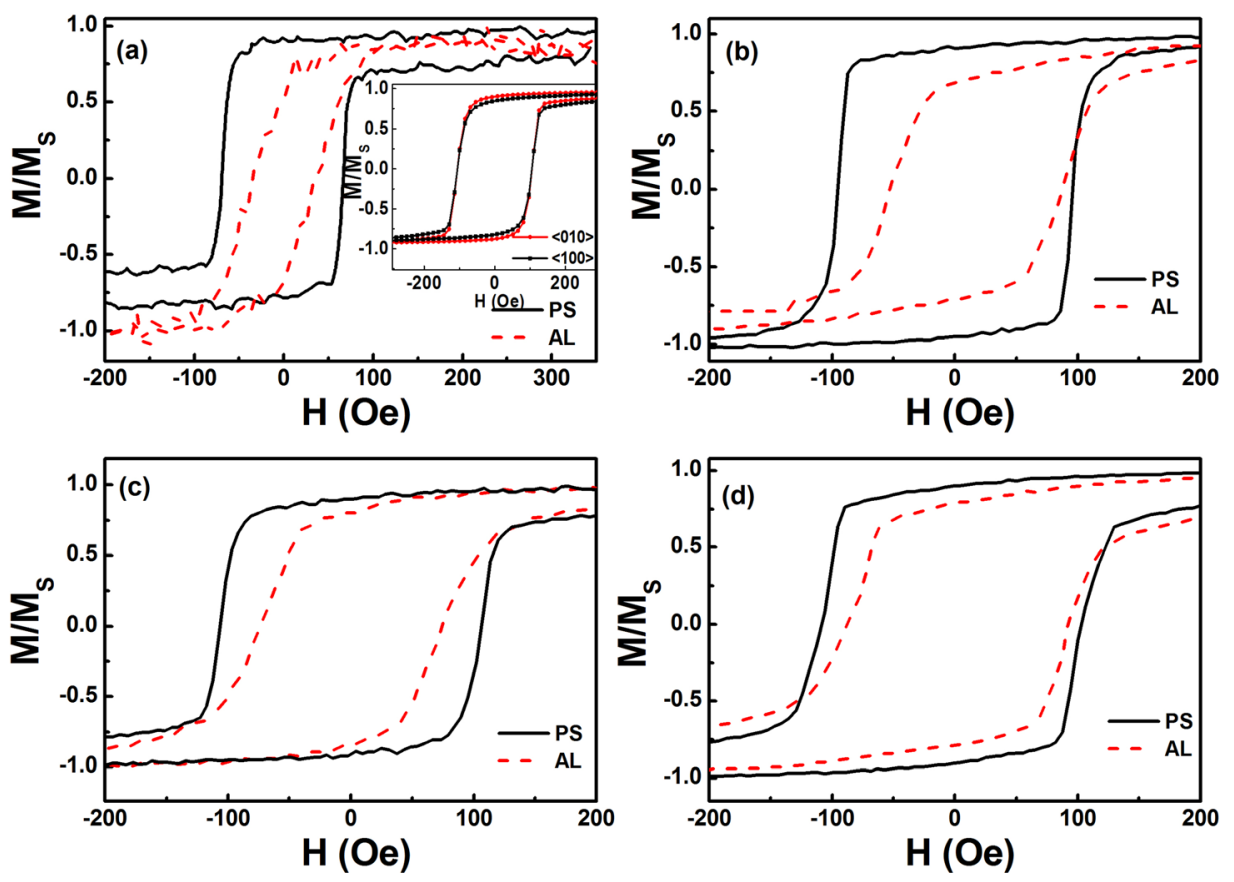

FIG. 2. M (H) loops measured at room temperature with the in-plane magnetic field applied along the steps (reddashed lines) and perpendicular to the steps (black-solid lines). The thicknesses of the magnetite films are $5 \mathrm{~nm}$ (a), $10 \mathrm{~nm}$ (b), $15 \mathrm{~nm}$ (c), and $20 \mathrm{~nm}$ (d), respectively. Inset: $\mathrm{M}$ (H) loops for $5 \mathrm{~nm}$ thick $\mathrm{Fe}_{3} \mathrm{O}_{4}$ film without steps with the field is applied along the $\langle 100\rangle$ and $\langle 010\rangle$ directions. very high chance of APB formation along step edges when growing $\mathrm{Fe}_{3} \mathrm{O}_{4}$ on stepped $\mathrm{MgO}$. Let us consider the alternately positioned A- and B-sites in the (100) atomic planes of $\mathrm{Fe}_{3} \mathrm{O}_{4}$. First, examining the case of nucleation at the B-sites, there are 32 possible combinations and 16 of them have the
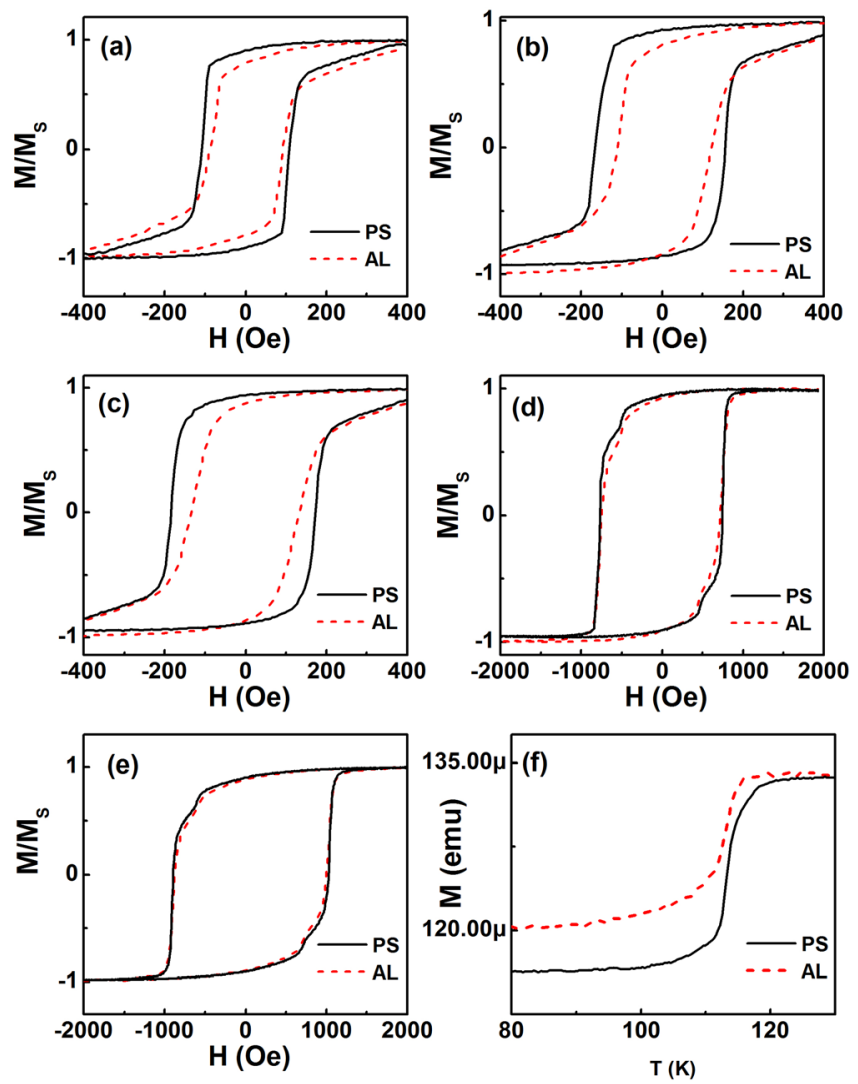

FIG. 3. M (H) loops with an in-plane magnetic field applied along the steps (red-dashed lines) and perpendicular to the steps (black-solid lines) measured at $300 \mathrm{~K}$ (a), $200 \mathrm{~K}$ (b), $150 \mathrm{~K}$ (c), $100 \mathrm{~K}$ (d), and $50 \mathrm{~K}$ (e), respectively. The thickness of the measured magnetite film is $20 \mathrm{~nm}$. (f) M-T curves measured at an in-plane field of 200 Oe applied along the steps (red-dashed lines) and perpendicular to the steps (black-solid lines). nucleation rows of the first B-site layer on the upper terrace parallel to the ones on the lower terrace which will result in the formation of APBs. The remaining 16 combinations have the rows of the first B site layer on the upper plane but perpendicular to the rows of the first B site layer on the lower plane. Of these 16 combinations, 8 of them will result in the formation of the APBs. Similarly, considering the second case when the nucleation starts at the A-sites, there are 64 possible combinations, but only 8 combinations do not result in the formation of APBs. Therefore, there is a high chance of forming APBs along the step edges. In other words, the high density of APBs formed along the step edges is seen to be responsible for orienting the easy axis perpendicular to the steps and hard axis along the steps edges.

It is well-known that APBs are responsible for the increased resistivity in magnetite films. ${ }^{36,42}$ If APB density along the step edges is higher than perpendicular to steps, the resistivity measured along the step edges should be higher than that measured perpendicular to steps. To confirm this, we studied the local transport properties of $60 \mathrm{~nm}$ thick stepped $\mathrm{Fe}_{3} \mathrm{O}_{4}$ films using sub- $100 \mathrm{~nm}$ nano-gap contacts. Two kinds of devices were fabricated: nano-gap contacts aligned along the steps (Fig. 4(a)) and nano-gap contacts placed perpendicular to the steps (Fig. 4(b)). The devices were fabricated by E-beam lithography (EBL) using positive tone resist, PMMA A3 supplied by MicroChem Corp. After development, thick metal contacts consisting of Ti $(5 \mathrm{~nm}) / \mathrm{Au}$ $(35 \mathrm{~nm})$ were deposited by e-beam evaporation. All the nanogap contacts were aligned either along the steps or perpendicular to steps. Subsequently, after lift-off with acetone, UV lithography was carried out to produce macroscopic metal contacts. Figure 4(c) shows the resistivity as a function of temperature (R-T) for the two kinds of devices. One can see from Fig. 4(c) that, above the Verwey transition temperature, the devices with nano-gap contacts placed along the steps have higher resistivity compared with those with nano-gap contacts placed perpendicular to the steps, which suggests that a high density of APBs are formed along the step edges. 

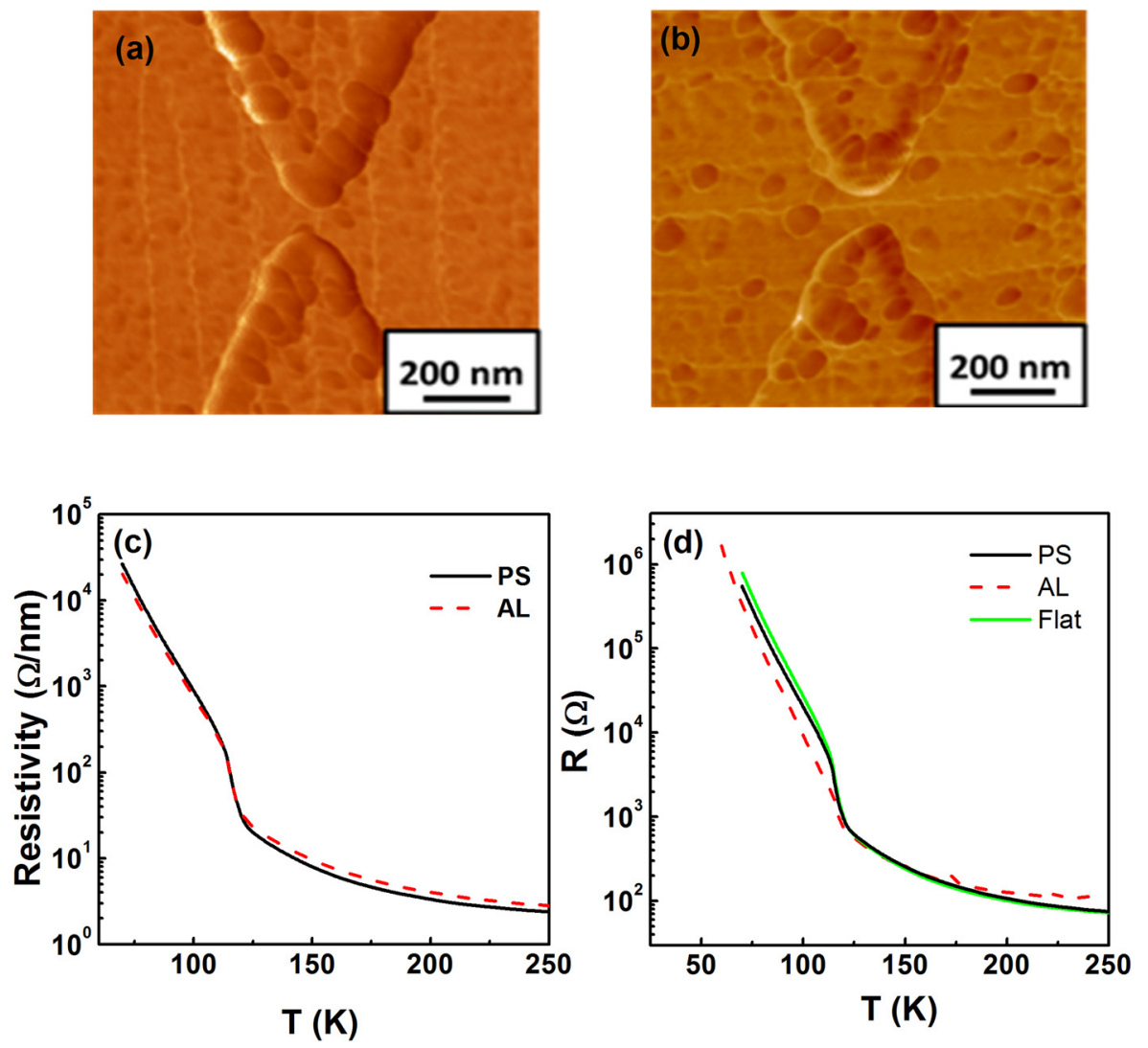

FIG. 4. $1 \times 1 \mu \mathrm{m}$ AFM images with a pair of nano-gap contacts placed (a) along the steps and (b) perpendicular to the steps. (c) Resistivity as a function of temperature for both configurations with nano-gap contacts. (d) Resistance as a function of temperature for both configurations with large scale contacts and for a flat $\mathrm{Fe}_{3} \mathrm{O}_{4}$ film with large scale contacts.
Interestingly, below the Verwey transition temperature, the devices with nano-gap contacts placed along the steps have a smaller resistivity compared with the devices with nano-gap contacts placed perpendicular to the steps. Thus, it is also noted the resistivity change across the Verwey transition temperature for the devices with nano-gap contacts placed perpendicular to the steps is greater than for the devices with nano-gap contacts placed along the steps, which suggests that APBs distort the formation of long range charge-ordering in magnetite. The temperature-dependent resistivity curves can be divided into three temperature regions, i.e., $\mathrm{T}>200 \mathrm{~K}$ (region I), $200 \mathrm{~K}<\mathrm{T}<120 \mathrm{~K}$ (region II), and $\mathrm{T}<120 \mathrm{~K}$ (region III), which correspond to three conduction mechanisms of magnetite. ${ }^{43} \mathrm{We}$ can fit the R-T curves in region II and region III with $R(T) \approx R_{0} \exp \left[\left(\frac{\mathrm{Ea}}{K_{B} T}\right)\right]$. Yielding activation energies $E_{a}$ for the two device configurations of $48 \mathrm{meV}$ (AL) and $52 \mathrm{meV}$ (PS) in region II and $65 \mathrm{meV}$ (AL) and $67 \mathrm{meV}$ (PS) in region III, respectively. The different activation energies for the two directions may also indicate that APBs distort long range charge-ordering formation in magnetite below and above the Verwey transition temperature. We also measured the same stepped $\mathrm{Fe}_{3} \mathrm{O}_{4}$ films with large scale contacts using the conventional four-probe method (Fig. 4(d)). The distance between the adjacent contacts is around $1 \mathrm{~mm}$. The R-T curves follow the same trend as the nano-gap contacts measurements since the steps are uniform, regular, and straight. To compare, we also measured a $60 \mathrm{~nm}$ flat $\mathrm{Fe}_{3} \mathrm{O}_{4}$ film with the same style of large scale contacts as for the stepped $\mathrm{Fe}_{3} \mathrm{O}_{4}$ film, with the current direction along the $\langle 001\rangle$ and $\langle 010\rangle$ directions. No difference in $\mathrm{R}-\mathrm{T}$ curves was observed for the orthogonal current directions. Therefore, only one R-T curve for flat $\mathrm{Fe}_{3} \mathrm{O}_{4}$ film is shown in Fig. 4(d). It can be clearly seen that the flat $\mathrm{Fe}_{3} \mathrm{O}_{4}$ film has a low resistance above the Verwey transition temperature and a high resistance below the Verwey transition temperature, which further indicates that APBs distort long range charge-ordering formation in magnetite.

In summary, we fabricated epitaxial stepped $\mathrm{Fe}_{3} \mathrm{O}_{4}$ thin films with different thicknesses and investigated their magnetic properties. Our magnetization measurements suggest that the steps induce additional anisotropy due to the high density of APBs formed at the step edges. Local transport measurements indicate that APBs distort the long range charge-ordering formation in magnetite.

This work was supported by Beijing Institute of Technology Research Fund Program for Young Scholars, Science Foundation of Ireland (SFI) under Contract No. 06/ IN.1/I91, National Plan for Science and technology (Nos. NPST 1598-02 and NPST 1466-02) of King Abdulaziz City for Science and Technology. H.C.W., MA.A., and MO.A. thank Saudi Aramco for the financial support (Project No. 6600028398). O.M. and A.S. acknowledge the financial support by the Bolashak Program funded by the Kazakhstan government.

${ }^{1}$ S. Sugahara and M. Tanaka, Appl. Phys. Lett. 80, 1969 (2002).

${ }^{2}$ Y. Z. Wu, C. Won, and Z. Q. Qiu, Phys. Rev. B 65, 184419 (2002).

${ }^{3}$ R. K. Kawakami, Ernesto J. Escorcia-Aparicio, and Z. Q. Qiu, Phys. Rev. Lett. 77, 2570 (1996).

${ }^{4}$ T. Leeb, M. Brockmann, F. Bensch, S. Miethaner, and G. Bayreuther, J. Appl. Phys. 85, 4964 (1999).

${ }^{5}$ J. Chen and J. L. Erskine, Phys. Rev. Lett. 68, 1212 (1992).

${ }^{6}$ H. J. Choi, Z. Q. Qiu, J. Pearson, J. S. Jiang, Dongqi Li, and S. D. Bader, Phys. Rev. B 57, R12713 (1998). 
${ }^{7}$ H. J. Elmers, J. Hauschild, and U. Gradmann, J. Magn. Magn. Mater. 221, 219 (2000).

${ }^{8}$ D. M. Engebreston, J. Berezovsky, J. P. Park, L. C. Chen, C. J. Palmstrom, and P. A. Crowell, J. Appl. Phys. 91, 8040 (2002).

${ }^{9}$ R. K. Kawakami, M. O. Bowen, H. J. Choi, E. J. Escorcia Aparicio, and Z. Q. Qiu, Phys. Rev. B 58, R5924 (1998).

${ }^{10}$ A. Berger, U. Linke, and H. P. Oepen, Phys. Rev. Lett. 68, 839 (1992).

${ }^{11}$ B. B. Maranville, A. L. Shapiro, and F. Hellman, Appl. Phys. Lett. 81, 517 (2002).

${ }^{12}$ S. K. Arora, B. J. O'Dowd, B. Ballesteros, P. Gambardella, and I. V. Shvets, Nanotech. 23, 235702 (2012).

${ }^{13}$ I. V. Shvets, H. C. Wu, V. Usov, F. Cuccureddu, S. K. Arora, and S. Murphy, Appl. Phys. Lett. 92, 023107 (2008).

${ }^{14}$ F. Walz, J. Phys.: Condens. Matter 14, R285 (2002).

${ }^{15}$ M. Ziese, Rep. Prog. Phys. 65, 143 (2002).

${ }^{16}$ J. H. V. J. Brabers, F. Walz, and H. Kronmuller, J. Phys.: Condens. Matter. 12, 5437 (2002).

${ }^{17}$ M. Monti, B. Santos, A. Mascaraque, O. Rodríguez de la Fuente, M. A. Niño, T. O. Menteş, A. Locatelli, K. F. McCarty, J. F. Marco, and J. de la Figuera, Phys. Rev. B 85, 020404 (2012).

${ }^{18}$ W. Q. Liu, Y. B. Xu, P. K. J. Wong, N. J. Maltby, S. P. Li, X. F. Wang, J. Du, B. You, J. Wu, P. Bencok, and R. Zhang, Appl. Phys. Lett. 104, 142407 (2014).

${ }^{19}$ S. Arora, H. C. Wu, R. Choudhary, I. Shvets, O. Mryasov, H. Yao, and W. Ching, Phys. Rev. B 77, 134443 (2008).

${ }^{20}$ R. Ramos, T. Kikkawa, K. Uchida, H. Adachi, I. Lucas, M. H. Aguirre, P. Algarabel, L. Morellon, S. Maekawa, E. Saitoh, and M. R. Ibarra, Appl. Phys. Lett. 102, 072413 (2013).

${ }^{21}$ Z. M. Liao, Y. D. Li, J. Xu, J. M. Zhang, K. Xia, and D. P. Yu, Nano Lett. 6, 1087 (2006).

${ }^{22}$ J. Gooth, R. Zierold, J. G. Gluschke, T. Boehnert, S. Edinger, S. Barth, and K. Nielsch, Appl. Phys. Lett. 102, 073112 (2013).

${ }^{23}$ S. Lee, A. Fursina, J. T. Mayo, C. T. Yavuz, V. L. Colvin, R. G. S. Sofin, I. V. Shvets, and D. Natelson, Nat. Mater. 7, 130 (2008).

${ }^{24}$ J. J. I. Wong, A. G. Swartz, R. J. Zheng, W. Han, and R. K. Kawakami, Phys. Rev. B 86, 060409 (2012).
${ }^{25}$ A. Fernández-Pacheco, J. M. De Teresa, J. Orna, L. Morellon, P. A. Algarabel, J. A. Pardo, M. R. Ibarra, C. Magen, and E. Snoeck, Phys. Rev. B 78, 212402 (2008).

${ }^{26}$ H. C. Wu, R. Ramos, R. G. S. Sofin, Z. M. Liao, M. Abid, and I. V. Shvets, Appl. Phys. Lett. 101, 052402 (2012).

${ }^{27}$ H. C. Wu, O. N. Mryasov, M. Abid, K. Radican, and I. V. Shvets, Sci. Rep. 3, 1830 (2013).

${ }^{28}$ X. Li, A. Gupta, G. Xiao, W. Qian, and V. Dravid, Appl. Phys. Lett. 73, 3282 (1998).

${ }^{29}$ H. C. Wu, S. K. Arora, O. N. Mryasov, and I. V. Shvets, Appl. Phys. Lett. 92, 182502 (2008).

${ }^{30}$ D. T. Margulies, F. T. Parker, M. L. Rudee, F. E. Spada, J. N. Chapman, P. R. Aitchison, and A. E. Berkowitz, Phys. Rev. Lett. 79, 5162 (1997).

${ }^{31}$ D. T. Margulies, F. T. Parker, F. E. Spada, R. S. Goldman, J. Li, R. Sinclair, and A. E. Berkowitz, Phys. Rev. B 53, 9175 (1996).

${ }^{32}$ W. Eerenstein, T. Hibma, and S. Celotto, Phys. Rev. B 70, 184404 (2004).

${ }^{33}$ F. C. Voogt, T. T. M. Palstra, L. Niesen, O. C. Rogojanu, M. A. James, and T. Hibma, Phys. Rev. B 57, R8107 (1998).

${ }^{34}$ S. K. Arora, R. G. S. Sofin, and I. V. Shvets, Phys. Rev. B 72, 134404 (2005).

${ }^{35}$ W. Eerenstein, T. T. M. Palstra, S. S. Saxena, and T. Hibma, Phys. Rev. Lett. 88, 247204 (2002).

${ }^{36}$ H. C. Wu, M. Abid, B. S. Chun, R. Ramos, O. N. Mryasov, and I. V. Shvets, Nano Lett. 10, 1132 (2010).

${ }^{37}$ W. B. Mi, J. J. Shen, E. Y. Jiang, and H. L. Bai, Acta Mater. 55, 1919 (2007).

${ }^{38}$ L. B. Zhao, W. B. Mi, E. Y. Jiang, and H. L. Bai, Appl. Phys. Lett. 91, 052113 (2007).

${ }^{39}$ M. S. Senn, J. P. Wright, and J. P. Attfield, Nature 481, 173 (2012).

${ }^{40}$ W. B. Mi, Z. B. Guo, Q. X. Wang, Y. Yang, and H. L. Bai, Scr. Mater. 68, 972 (2013).

${ }^{41}$ R. G. S. Sofin, H. C. Wu, and I. V. Shvets, Phys. Rev. B 84, 212403 (2011).

${ }^{42}$ W. Eerenstein, T. T. M. Palstra, T. Hibma, and S. Celotto, Phys. Rev. B 66, R201101 (2002)

${ }^{43}$ R. Ramos, S. K. Arora, and I. V. Shvets, Phys. Rev. B 78, 214402 (2008). 